\title{
Serum levels of sclerostin as a potential biomarker in central arterial stiffness among hypertensive patients
}

\author{
Yu-Chi Chang ${ }^{1 \dagger}$, Bang-Gee Hsu ${ }^{1,2+}$, Hung-Hsiang Liou ${ }^{3}$, Chung-Jen Lee ${ }^{4}$ and Ji-Hung Wang ${ }^{1,5^{*}}$ (D)
}

\begin{abstract}
Background: Sclerostin is known to be a canonical Wnt/B-catenin signaling pathway inhibitor, while the Wnt/ $\beta$ catenin signaling pathway is proposed to be involved in the development of arterial stiffness. This study aims to investigate the relationship between serum sclerostin levels and carotid-femoral pulse wave velocity (cfPWV) among hypertensive patients.

Methods: Fasting blood samples were obtained from 105 hypertensive patients. Patients with cfPWV values of $>10 \mathrm{~m} / \mathrm{s}$ were classified in the high arterial stiffness group, whereas those with cfPWV values of $\leq 10 \mathrm{~m} / \mathrm{s}$ were assigned to the low arterial stiffness group. Serum sclerostin and Dickkopf-1 (DKK1) levels were quantified using commercially available enzyme-linked immunosorbent assays.
\end{abstract}

Results: Thirty-six hypertensive patients (34.3\%) who belonged to the high arterial stiffness group were generally older ( $p<0.001$ ), presented with lower estimated glomerular filtration rates (eGFR, $p=0.014$ ), higher incidence of diabetes mellitus ( $p=0.030$ ), average systolic blood pressures (SBP, $p=0.013$ ), pulse pressure $(p=0.026)$, serum creatinine levels $(p=0.013$ ), intact parathyroid hormone levels (iPTH, $p=0.003$ ), and sclerostin levels $(p<0.001)$ than their counterparts in the low arterial stiffness group. A multivariable logistic regression analysis identified sclerostin as an independent predictor of arterial stiffness in hypertensive patients (odds ratio, 1.042; 95\% confidence interval $(C I), 1.017-1.068 ; p=0.001)$. Multivariable forward stepwise linear regression analysis also showed that serum sclerostin level ( $\beta=0.255$, adjusted $R^{2}$ change: $0.146, p=0.003$ ) was positively associated with cfPW values in patients with hypertension.

Conclusions: In this study, serum sclerostin level, but not DKK1, is found to be positively correlated with cfPWW values and is identified as an independent predictor of arterial stiffness in hypertensive patients after adjusting for significant confounders.

Keywords: Arterial stiffness, Carotid-femoral pulse wave velocity, Hypertension, Sclerostin, Dickkopf-1

\section{Background}

Hypertension is a primary risk factor for morbidity and mortality, which leads to the development of atherosclerosis and target organ damage such as cerebral vascular events, retinopathy, and heart failure [1]. Several studies have reported arterial stiffness to be closely related to cardiovascular morbidity and mortality in hypertensive patients [2-4]. These evidences seem to point towards the

\footnotetext{
* Correspondence: abanggeelily@gmail.com

${ }^{\dagger}$ Yu-Chi Chang and Bang-Gee Hsu contributed equally to this work.

'School of Medicine, Tzu Chi University, Hualien, Taiwan

${ }^{5}$ Division of Cardiology, Buddhist Tzu Chi General Hospital, No. 707, Section

3, Chung-Yang Road, Hualien 97004, Taiwan

Full list of author information is available at the end of the article
}

hypothesis that central arterial stiffness is in fact a precursor rather than a sequela of hypertension [5].

Arterial stiffness, which results from progressive breakdown of elastic fibers in the aorta and large elastic arteries, causes increased pulse wave velocity (PWV), decreased diastolic coronary perfusion pressure, and will eventually lead to diminished myocardial oxygen delivery $[6,7]$. Carotid-femoral PWV (cfPWV), calculated as the time required for arterial pulse to spread from the carotid to the femoral artery, can be used to measure central arterial stiffness [8]. Today, it is not only the gold standard to assess central arterial stiffness but also an

(c) The Author(s). 2018 Open Access This article is distributed under the terms of the Creative Commons Attribution 4.0 International License (http://creativecommons.org/licenses/by/4.0/), which permits unrestricted use, distribution, and reproduction in any medium, provided you give appropriate credit to the original author(s) and the source, provide a link to the Creative Commons license, and indicate if changes were made. The Creative Commons Public Domain Dedication waiver (http://creativecommons.org/publicdomain/zero/1.0/) applies to the data made available in this article, unless otherwise stated. 
independent predictor of the outcome of well-recognized cardiovascular events $[3,9]$. Moreover, cfPWV has been recommended as a tool for the diagnosis of target organ damage in patients with earlier stages of hypertension [10].

When a Wnt ligand binds with its low-density lipoprotein receptor-related protein (LRP5/6) and Frizzled co-receptors on the cell surface of osteoblasts, intracellular $\beta$-catenin degradation is decreased, and this $\beta$-catenin then triggers targeted gene transcription to regulate bone turnover [11]. High-phosphate environment activates the $\mathrm{Wnt} / \beta$-catenin signaling pathway and promotes osteogenic transdifferentiation and calcification of vascular smooth muscle cells through direct modulation of Runx2 gene expression [12]. The Wnt/ $\beta$-catenin pathway mediates oxidized low-density lipoprotein-induced endothelial injury via oxidative stress and it plays an important role in the procession and development of atherosclerosis [13]. Another study noted that the Wnt/ $\beta$-catenin signaling pathway is the master upstream regulator which controls the expression of multiple intrarenal renin-angiotensin system genes and plays an essential role in the pathogenesis of hypertension [14]. Thus, we can see that the Wnt/ $\beta$-catenin signaling pathway contributes to the development of vascular calcification, atherosclerosis and hypertension. Sclerostin and Dickkopf-1 (DKK1) interact with the extracellular domains on LRP5 and LRP6 to competitively prevent the binding of various Wnt ligands to these coreceptors and is a typical Wnt/ $\beta$-catenin signaling pathway inhibitor $[15,16]$. More recently, studies showed that the expression of $\mathrm{Wnt} / \beta$-catenin signaling inhibitors such as sclerostin and DKK1 may avoid further vascular calcification [17, 18]. However, the relationship between central arterial stiffness and serum levels of sclerostin and DKK1 among hypertensive patients remains unclear. In this study, we aimed to investigate this association in patients with hypertension.

\section{Methods \\ Patients}

Between January 2012 and April 2012, 115 hypertensive patients were recruited in the cardiovascular outpatient department (Dr. Ji-Hung Wang) of a medical center located in Hualien, Taiwan. Among the initial 115 hypertensive participants, 10 were excluded on account of acute infection $(n=1)$, acute myocardial infarction $(n=1)$, arrhythmia $(n=1)$, pulmonary edema $(n=1)$, or refusal to provide informed consents $(n=6)$. In the end, a total of 105 hypertensive participants, 69 men and 36 women, were included in the study. The Protection of the Human Subjects Institutional Review Board of Tzu Chi University and Hospital approved this study. All participants provided their written informed consents to take part in this study. Trained staff measured the morning blood pressures (BP) of our participants who have rested for at least $10 \mathrm{~min}$ using standard mercury sphygmomanometers with appropriate cuff sizes. Systolic BP (SBP) and diastolic BP (DBP) values were recorded at the points of appearance and disappearance of the Korotkoff sounds, respectively. SBP and DBP values were measured three times at 5 -min intervals and were averaged for the analysis. For the prevalence survey, hypertension was defined as $\mathrm{SBP} \geq 140$ $\mathrm{mmHg}$ and/or $\mathrm{DBP} \geq 90 \mathrm{mmHg}$, or patients who were taking antihypertensive drugs. Diabetes mellitus (DM) were diagnosed as patients whose fasting plasma glucose levels $\geq 126 \mathrm{mg} / \mathrm{dL}(6.1 \mathrm{mmol} / \mathrm{L})$ or those who were using hypoglycemic agents [19].

\section{Anthropometric measurements}

We measured the body weight of our patients wearing light clothing and without shoes, rounded to the nearest $0.5 \mathrm{~kg}$; and height to the nearest $0.5 \mathrm{~cm}$. We calculated the body mass indexes using the formulae of the weight in kilograms divided by the height in squared meters [20-22].

\section{Biochemical investigations}

Fasting blood samples (approximately $5 \mathrm{~mL}$ ) were immediately centrifuged at $3000 \mathrm{~g}$ for $10 \mathrm{~min}$. Serum levels of blood urea nitrogen (BUN), creatinine, fasting glucose, total cholesterol (TCH), triglycerides (TG), high-density lipoprotein cholesterol (HDL-C), low-density lipoprotein cholesterol (LDL-C), total calcium, and phosphorus were measured using an autoanalyzer (COBAS Integra 800, Roche Diagnostics, Basel, Switzerland) [20-22]. We used commercially available enzyme-linked immunosorbent assays to measure the levels of serum sclerostin, DKK1 (Biomedica Immunoassays, Vienna, Austria) and intact parathyroid hormone (iPTH, Diagnostic Systems Laboratories, Texas, USA) [20]. We also calculated the estimated glomerular filtration rate (eGFR) using the CKD-EPI (Chronic Kidney Disease Epidemiology Collaboration) equation.

\section{Carotid-femoral pulse wave velocity measurements}

The same observer performed the measurements of cfPWV values to record the pressure pulse waveform in the underlying artery [21, 22], by using a pressure tonometer (SphygmoCor system, AtCor Medical, Sydney, New South Wales, Australia). All measurements were performed in the morning with patients in supine position after a minimum of 10 -min rest in a quiet, temperature-controlled room. The patients were required to abstain from smoking and consuming alcohol or coffee prior to the procedure. Records were made simultaneously with an ECG signal, which provided an $R$-timing reference. Pulse wave recordings were performed consecutively at two superficial artery sites 
(carotid-femoral segment). Integral software was used to process each set of pulse wave and ECG data to calculate the mean time difference between the $R$-wave and pulse wave on a beat-to-beat basis, with an average of 10 consecutive cardiac cycles. The cfPWV was calculated using the distance and mean time difference between the two recorded points. Quality indices, included in the software, were set to ensure uniformity of the data. In this study, patients with cfPWV values $>10 \mathrm{~m} / \mathrm{s}$ were assigned to the high arterial stiffness group, whereas those with values $\leq 10 \mathrm{~m} / \mathrm{s}$ constituted the low arterial stiffness group according to the ESH-ESC 2013 guidelines [10].

\section{Statistical analysis}

Normally distributed data were expressed as mean \pm standard deviation, and comparisons between patients were performed using the Student's independent $t$-test (two-tailed). Data that were not normally distributed were expressed as median and interquartile ranges; while the Mann-Whitney $\mathrm{U}$ test was used for the comparisons of TG, fasting glucose, and DKK1 levels between patients. Categorical variables (expressed by numbers of patients) were analyzed by the $x^{2}$ test. Variables that were significantly associated with arterial stiffness in hypertensive patients were tested for independence using a forward multivariable logistic regression analysis (adapted factors: DM, age, SBP, eGFR, iPTH, and sclerostin). Since serum TG, fasting glucose, and DKK1 levels were not normally distributed; therefore, logarithmic transformations were applied to achieve normality. Correlation between clinical variables and cfPWV values in patients with hypertension was evaluated using a simple linear regression analysis, and variables that were significantly correlated with cfPWV values were tested for independence using a multivariable forward stepwise regression analysis (adopted factors: DM, age, SBP, HDL-C, BUN, creatinine, eGFR, iPTH, and sclerostin). Data were analyzed using the SPSS software for Windows (version 19.0; SPSS, Chicago, IL, USA). A $p$ value $<0.05$ was considered as statistically significant.

\section{Results}

Demographic, biochemical, and clinical characteristics of the 105 hypertensive patients are shown in Table 1 . The medical histories included diabetes mellitus $(n=46$; $43.8 \%)$ and dyslipidemia $(n=87 ; 82.9 \%)$. The medications used included angiotensin-converting enzyme inhibitor (ACEI; $n=33 ; 31.4 \%$ ), angiotensin receptor blocker (ARB; $n=60 ; 57.1 \%), \beta$-blocker $(n=62 ; 59.0 \%)$, calcium channel blocker (CCB; $n=49 ; 46.7 \%)$, statin ( $n$ $=64 ; 61.0 \%)$, and fibrate $(n=17 ; 16.2 \%)$. Thirty-six hypertensive patients (34.3\%) were included in the high arterial stiffness group. More hypertensive patients who had DM were in the high arterial stiffness group ( $p=$ 0.030). No statistically significant differences were observed in gender, serum DKK1 levels, ACEI, ARB, $\beta$-blocker, CCB, statin, or fibrate use between the two arterial stiffness groups. The patients in the high arterial stiffness group presented older age $(p<0.001)$, higher $\operatorname{SBP}(p=0.013)$, serum creatinine $(p=0.013)$, iPTH $(p=$ $0.003)$, and sclerostin levels $(p<0.001)$, and lower eGFR $(p=0.014)$ than the patients in the low arterial stiffness group.

Our forward multivariable logistic regression analysis, after adjusting for the factors significantly associated with arterial stiffness (diabetes mellitus, age, SBP, eGFR, iPTH, and sclerostin) showed that sclerostin (odds ratio $=1.042$; $95 \% \mathrm{CI}=1.017-1.068 ; p=0.001$ ), age (odds ratio $=1.088$, $95 \% \mathrm{CI}=1.021-1.159, p=0.010$ ), and $\mathrm{iPTH}$ (odds ratio $=1.025,95 \% \mathrm{CI}=1.003-1.048, p=0.026$ ) were independent predictors of arterial stiffness in hypertensive patients (Table 2).

The correlation between cfPWV values and clinical variables among patients with hypertension is shown in Table 3. Simple linear regression analysis found DM $(r=$ $0.228, p=0.019)$, age $(r=0.366, p<0.001)$, SBP $(r=$ $0.334, p=0.001)$, BUN $(r=0.243, p=0.013)$, creatinine $(r=0.369, p<0.001)$, iPTH $(r=0.291, p=0.003)$, and sclerostin levels $(r=0.392, p<0.001)$ to be positively correlated with cfPWV values, whereas HDL-C $(r=-0.267$, $p=0.006)$, eGFR $(r=-0.335, p<0.001)$ was negatively correlated with cfPWV values. Log-DKK1 levels showed no significant correlation with cfPWV values. Multivariable forward stepwise linear regression analysis revealed that age $\left(\beta=0.325\right.$, adjusted $R^{2}$ change $=0.109, p<$ $0.001)$, SBP $\left(\beta=0.236\right.$, adjusted $R^{2}$ change $=0.061, p=$ $0.004)$, iPTH $\left(\beta=0.185\right.$, adjusted $R^{2}$ change $=0.032, p=$ $0.022)$, HDL-C ( $\beta=-0.193$, adjusted $R^{2}$ change $=0.025$, $p=0.027)$, and sclerostin levels $\left(\beta=0.255\right.$, adjusted $R^{2}$ change $=0.146, p=0.003$ ) were independent predictors of cfPWV values in our patients with hypertension. Two-dimensional scattered plots of serum sclerostin levels and cfPWV values among the 105 hypertensive patients are shown in Fig. 1.

\section{Discussion}

This study demonstrated that serum sclerostin levels were higher in hypertensive patients who had central arterial stiffness. Together with other confounders, serum sclerostin levels were also an independent marker of cfPWV values. However, serum DKK1 levels were not different among hypertensive patients by this measure of central arterial stiffness.

The aging process of blood vessel is primarily associated with changes in the arterial structure, thickening, and loss of elasticity over time, resulting in an increase in arterial stiffness [23]. Vascular calcification is associated with 
Table 1 Clinical variables of the 105 hypertensive patients with or without arterial stiffness

\begin{tabular}{|c|c|c|c|c|}
\hline Characteristic & $\begin{array}{l}\text { All participants } \\
(n=105)\end{array}$ & $\begin{array}{l}\text { Low AS group } \\
(n=69)\end{array}$ & $\begin{array}{l}\text { High AS group } \\
(n=36)\end{array}$ & $p$ value \\
\hline Age (years) & $64.51 \pm 10.21$ & $61.96 \pm 10.01$ & $69.42 \pm 8.80$ & $<0.001^{*}$ \\
\hline Height (cm) & $160.92 \pm 8.47$ & $161.55 \pm 8.45$ & $159.72 \pm 8.50$ & 0.296 \\
\hline Body weight (kg) & $69.12 \pm 12.31$ & $68.71 \pm 13.38$ & $69.91 \pm 10.10$ & 0.639 \\
\hline Body mass index $\left(\mathrm{kg} / \mathrm{m}^{2}\right)$ & $26.60 \pm 3.61$ & $26.16 \pm 3.61$ & $27.43 \pm 3.50$ & 0.088 \\
\hline $\operatorname{cfPWV}(\mathrm{m} / \mathrm{s})$ & $9.33 \pm 2.76$ & $7.78 \pm 1.43$ & $12.29 \pm 2.23$ & $<0.001^{*}$ \\
\hline Systolic blood pressure (mmHg) & $134.52 \pm 17.85$ & $131.41 \pm 14.94$ & $140.50 \pm 21.39$ & $0.013^{*}$ \\
\hline Diastolic blood pressure (mmHg) & $73.72 \pm 9.95$ & $73.23 \pm 9.96$ & $74.67 \pm 10.01$ & 0.486 \\
\hline Total cholesterol (mg/dL) & $174.51 \pm 38.22$ & $176.94 \pm 36.85$ & $169.86 \pm 40.84$ & 0.370 \\
\hline Triglyceride (mg/dL) & $121.00(93.50-172.00)$ & $121.00(94.00-174.00)$ & $128.00(89.50-173.50)$ & 0.718 \\
\hline $\mathrm{HDL}-\mathrm{C}(\mathrm{mg} / \mathrm{dL})$ & $45.62 \pm 13.37$ & $46.72 \pm 13.86$ & $43.50 \pm 12.28$ & 0.243 \\
\hline $\mathrm{LDL}-\mathrm{C}(\mathrm{mg} / \mathrm{dL})$ & $103.25 \pm 28.92$ & $103.96 \pm 26.20$ & $101.89 \pm 33.89$ & 0.730 \\
\hline Fasting glucose (mg/dL) & $114.00(98.50-157.00)$ & $116.00(98.50-158.00)$ & $115.00(97.75-153.75)$ & 0.893 \\
\hline Blood urea nitrogen (mg/dL) & $16.91 \pm 5.26$ & $16.20 \pm 4.74$ & $18.28 \pm 5.97$ & 0.054 \\
\hline Creatinine (mg/dL) & $1.10 \pm 0.33$ & $1.06 \pm 0.28$ & $1.19 \pm 0.41$ & $0.042^{*}$ \\
\hline eGFR (mL/min) & $69.34 \pm 20.51$ & $72.86 \pm 19.07$ & $62.60 \pm 21.72$ & $0.014^{*}$ \\
\hline Total calcium (mg/dL) & $9.17 \pm 0.37$ & $9.19 \pm 0.37$ & $9.14 \pm 0.36$ & 0.512 \\
\hline Phosphorus (mg/dL) & $3.52 \pm 0.51$ & $3.56 \pm 0.52$ & $3.43 \pm 0.49$ & 0.219 \\
\hline iPTH (pg/mL) & $50.81 \pm 27.71$ & $45.12 \pm 22.04$ & $61.72 \pm 33.94$ & $0.003^{*}$ \\
\hline Sclerostin (pmol/L) & $69.34 \pm 20.51$ & $52.58 \pm 21.66$ & $74.77 \pm 31.02$ & $<0.001^{*}$ \\
\hline Dickkopf-1 (pmol/L) & $14.00(6.06-28.82)$ & $14.61(5.94-30.23)$ & $13.52(6.02-25.51)$ & 0.845 \\
\hline Female, $n(\%)$ & $36(34.3)$ & $25(36.2)$ & $11(30.6)$ & 0.561 \\
\hline Diabetes mellitus, $n(\%)$ & $46(43.8)$ & $25(36.2)$ & $21(58.3)$ & $0.030^{*}$ \\
\hline ACE inhibitor, $n(\%)$ & $33(31.4)$ & $22(31.9)$ & $11(30.6)$ & 0.889 \\
\hline Angiotensin receptor blocker, $n(\%)$ & $60(57.1)$ & $39(56.5)$ & $21(58.3)$ & 0.859 \\
\hline$\beta$-blocker, $n$ (\%) & $62(59.0)$ & 37 (53.6) & $25(69.4)$ & 0.118 \\
\hline Calcium channel blocker, $n$ (\%) & $49(46.7)$ & $29(42.0)$ & $20(55.6)$ & 0.187 \\
\hline Statin, $n(\%)$ & $64(61.0)$ & $46(66.7)$ & $18(50.0)$ & 0.097 \\
\hline Fibrate, $n(\%)$ & $17(16.2)$ & $8(11.6)$ & $9(25.0)$ & 0.077 \\
\hline
\end{tabular}

Values for continuous variables are shown as mean \pm standard deviation after analysis by Student's $t$-test; variables not normally distributed are shown as median and interquartile range after analysis by the Mann-Whitney $\mathrm{U}$ test; values are presented as number (\%) and analysis after analysis by the chi-square test AS arterial stiffness, cfPWV carotid-femoral pulse wave velocity, HDL-C high-density lipoprotein cholesterol, LDL-C low-density lipoprotein cholesterol, eGFR estimated glomerular filtration rate, $i P T H$ intact parathyroid hormone, ACE angiotensin-converting enzyme

Patients with cfPWV values $>10 \mathrm{~m} / \mathrm{s}$ were assigned to the high AS group, whereas those with values $\leq 10 \mathrm{~m} / \mathrm{s}$ constituted the low AS group

*Values of $p<0.05$ were considered statistically significant

Table 2 Multivariable logistic regression analysis of the factors correlated with arterial stiffness among the 105 hypertensive patients

\begin{tabular}{llll}
\hline Variables & Odds ratio & $95 \%$ confidence interval & $p$ value \\
\hline Sclerostin (pmol/L) & 1.042 & $1.017-1.068$ & $0.001^{*}$ \\
Age & 1.088 & $1.021-1.159$ & $0.010^{*}$ \\
Intact parathyroid hormone & 1.025 & $1.003-1.048$ & $0.026^{*}$ \\
Systolic blood pressure & 1.028 & $0.996-1.062$ & 0.086 \\
Diabetes mellitus & 2.189 & $0.769-6.235$ & 0.142 \\
Estimated glomerular filtration rate & 0.996 & $0.969-1.024$ & 0.794 \\
\hline
\end{tabular}

Patients with carotid-femoral pulse wave velocity values $>10 \mathrm{~m} / \mathrm{s}$ were assigned to the arterial stiffness in this study

*Values of $p<0.05$ were considered statistically significant in the forward multivariate logistic regression analysis (adopted factors: diabetes mellitus, age, systolic blood pressure, estimated glomerular filtration rate, intact parathyroid hormone, and sclerostin) 
Table 3 Correlation between carotid-femoral pulse wave velocity levels and clinical variables among the 105 hypertensive patients

\begin{tabular}{|c|c|c|c|c|c|}
\hline \multirow[t]{3}{*}{ Variables } & \multicolumn{5}{|c|}{ Carotid-femoral pulse wave velocity $(\mathrm{m} / \mathrm{s})$} \\
\hline & \multicolumn{2}{|c|}{ Simple linear regression } & \multicolumn{3}{|c|}{ Multivariable linear regression } \\
\hline & $\bar{r}$ & $p$ value & Beta & Adjusted $R^{2}$ change & $p$ value \\
\hline Female & -0.154 & 0.116 & - & - & - \\
\hline Diabetes mellitus & 0.228 & $0.019^{*}$ & - & - & - \\
\hline Age (years) & 0.366 & $<0.001^{*}$ & 0.325 & 0.109 & $<0.001^{*}$ \\
\hline Height (cm) & -0.017 & 0.866 & - & - & - \\
\hline Body weight $(\mathrm{kg})$ & -0.003 & 0.975 & - & - & - \\
\hline Body mass index $\left(\mathrm{kg} / \mathrm{m}^{2}\right)$ & 0.025 & 0.800 & - & - & - \\
\hline Systolic blood pressure (mmHg) & 0.334 & $0.001^{*}$ & 0.236 & 0.061 & $0.004^{*}$ \\
\hline Diastolic blood pressure $(\mathrm{mmHg})$ & 0.100 & 0.308 & - & - & - \\
\hline Total cholesterol (mg/dL) & -0.110 & 0.263 & - & - & - \\
\hline Log-Triglyceride (mg/dL) & -0.001 & 0.991 & - & - & - \\
\hline $\mathrm{HDL}-\mathrm{C}(\mathrm{mg} / \mathrm{dL})$ & -0.267 & $0.006^{*}$ & -0.193 & 0.025 & $0.027^{*}$ \\
\hline $\mathrm{LDL}-\mathrm{C}(\mathrm{mg} / \mathrm{dL})$ & -0.019 & 0.849 & - & - & - \\
\hline Log-Glucose (mg/dL) & 0.001 & 0.998 & - & - & - \\
\hline Blood urea nitrogen (mg/dL) & 0.243 & $0.013^{*}$ & - & - & - \\
\hline Creatinine $(\mathrm{mg} / \mathrm{dL})$ & 0.369 & $<0.001^{*}$ & - & - & - \\
\hline eGFR (mL/min) & -0.335 & $<0.001^{*}$ & - & - & - \\
\hline Total calcium (mg/dL) & -0.027 & 0.785 & - & - & - \\
\hline Phosphorus (mg/dL) & -0.101 & 0.306 & - & - & - \\
\hline iPTH (pg/mL) & 0.293 & $0.003^{*}$ & 0.185 & 0.032 & $0.022^{*}$ \\
\hline Sclerostin (pmol/L) & 0.392 & $<0.001^{*}$ & 0.255 & 0.146 & $0.003^{*}$ \\
\hline Log-Dickkopf-1 (pmol/L) & -0.250 & 0.142 & - & - & - \\
\hline
\end{tabular}

Data of triglyceride, glucose, and dickkopf-1 showed skewed distribution and therefore were log-transformed before analysis

Analysis of data was done using the simple linear regression analyses or multivariable stepwise linear regression analysis (adapted factors were diabetes mellitus, age, systolic blood pressure, HDL-C, blood urea nitrogen, creatinine, eGFR, iPTH, and sclerostin)

$H D L-C$ high-density lipoprotein cholesterol, LDL-C low-density lipoprotein cholesterol, eGFR estimated glomerular filtration rate, iPTH intact parathyroid hormone *Values of $p<0.05$ were considered statistically significant

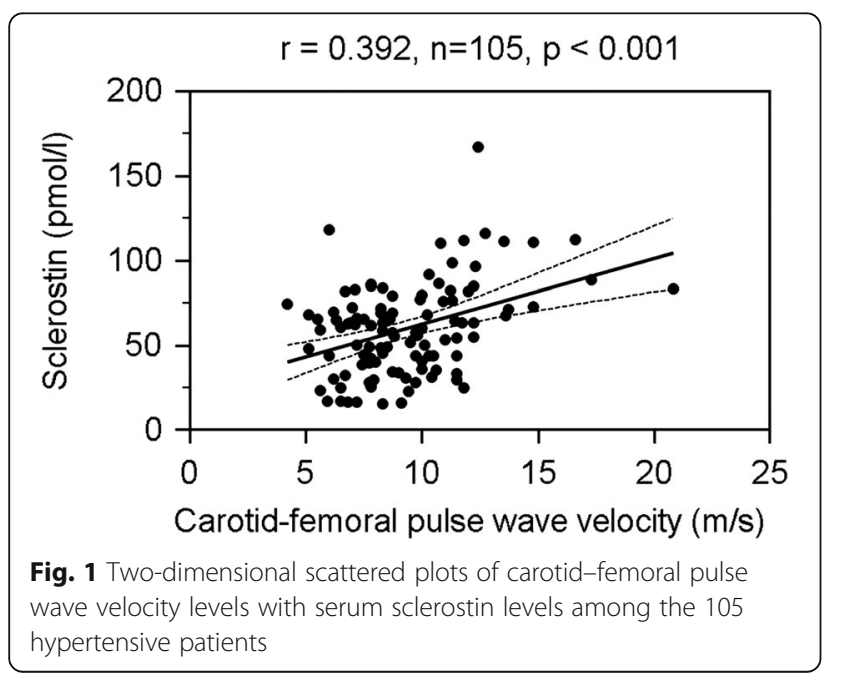

central arterial stiffness which leads to earlier reflected pressure waves from the arterioles toward the heart, causing an elevation in SBP and pulse pressure [7]. Patients with impaired glucose metabolism and type 2 DM were reported to have high central arterial stiffness [24]. One systematic review found that in approximately $90 \%$ of the studies, age and BP were consistently independently associated with cfPWV values, whereas DM was associated with cfPWV values in $52 \%$ of the studies [25]. A study found that BP was positively associated with the cfPWV values and was regarded as the main driver of arterial stiffening in 1052 untreated patients [26]. Similarly, we found that hypertensive patients with high arterial stiffness were older, presented with higher SBPs, and had a higher incidence of DM. Furthermore, we found older age to be the risk factors for the development of arterial stiffness after adjusting the covariates. Age and SBP were also positively associated with cfPWV values in patients with hypertension. 
Central arterial stiffness transfers excessive flow pulsatility into the renal microvasculature, leading to dynamic constriction or vessel loss that contributes to lower eGFR [27]. The aortic PWV value was shown to be negatively associated with eGFR values in 2564 patients from the Chronic Renal Insufficiency Cohort (CRIC) study [28]. However, conflicting result regarding cfPWV values and eGFR was found in another clinical study [29], probably due to the differences in the number of CKD population and various disease comorbidities. Our results showed that hypertensive patients with high arterial stiffness had higher serum creatinine and lower eGFR values. HDL-C can both have anti-atheromatous effects on the arterial wall and protective effects on endothelial cell, through which may improve arterial stiffness [30, 31]. Our results showed HDL-C levels were negatively associated with cfPWV values in patients with hypertension. A similar finding was also observed in community-dwelling individuals and healthy adults in China [32, 33]. Elevation in serum parathyroid hormone (PTH) often accompanies renal impairment [34]. PTH was associated with an increased risk of nonfatal atherosclerotic disease in two prospective, community-based studies focusing on elderly patients [35]. Moreover, a recent study found that PTH had both a direct and a BP-mediated indirect effect on the cfPWV values [26]. In our study, elevated iPTH levels revealed a significant association with high arterial stiffness and iPTH levels was also positively associated with cfPWV values in patients with hypertension.

Vascular calcification is associated with arterial stiffness and osteoprogenitor cells play an important role in the bone-vascular axis which regulates calcium metabolism around the arterial wall [36, 37]. Many factors can modulate vascular calcification, such as osteoblastic differentiation, vitamin D status, matrix Gla protein or even oxidative stress [16]. However, vascular calcification physiopathology is still poorly understood [16]. One of the mechanism that affects osteoblastic differentiation and contributes to the development of vascular calcification is the $\mathrm{Wnt} / \beta$-catenin signaling pathway $[17,18]$. Sclerostin is a signaling pathway inhibitor of the Wnt/ $\beta$-catenin pathway that contributes to reduced bone turnover and upregulates the calcification induced by vascular smooth muscle cells $[15,16,38,39]$. After vascular calcification has progressed, serum sclerostin is elevated to decrease $\beta$-catenin stability in the cells and suppress the proliferation and differentiation of osteoprogenitor cells $[15,16,37]$. Clinical studies have reported that serum sclerostin levels are positively correlated with carotid intima-media thickness in hemodialysis patients, and positively associated with coronary artery or abdominal aortic calcification in non-dialysis CKD patients [40-42]. Moreover, serum sclerostin levels were found to be positively associated with brachial-ankle PWV values in kidney transplantation patients, and positively associated with cfPWV values in chronic kidney disease (CKD), hemodialysis, and postmenopausal women [20, 43-45]. In type 2 DM patients with atherosclerotic lesions, serum sclerostin level is also increased [46]. Accordingly, our study also showed that serum sclerostin levels were identified as independent predictors of central arterial stiffness in hypertensive patients. DKK1 is another inhibitor of the Wnt $/ \beta$-catenin pathway [47]. However, some studies did not find the associated with arterial stiffness. Serum DKK1 levels were not found to be associated with brachial-ankle PWV values in kidney transplantation patients [20]. Another study noted plasma DKK1 levels did not associate with PWV in postmenopausal women [45]. In this study, we did not find the association between DKK1 and central arterial stiffness in hypertensive patients. Further studies are required to elucidate the relationship between central arterial stiffness and serum DKK1 level in hypertensive patients.

The limitations of our study include its cross-sectional design and the limited number of participants enrolled, which do not allow for exclusion of bias. Also, we are aware that antihypertensive medications, dyslipidemia, $\mathrm{DM}$, and lifestyle modifications may also affect cfPWV values. In addition, we did not assess vascular endothelial function through flow-mediated dilatation or inflammation markers such as $\mathrm{C}$-reactive protein and vitamin D status.

Despite contradictory findings that showed serum sclerostin levels to be positively correlated with carotid intima-media thickness and inversely correlated with cfPWV values in 67 subjects at Catania, Italy [48], it may be explained by the differences in ethnicity of populations, inclusion or exclusion criteria, race, or locations between these two studies. Additional long-term prospective studies or randomized controlled trials are needed to confirm our finding that sclerostin levels can predict central arterial stiffness among hypertensive patients.

\section{Conclusions}

For the inhibitors of the $\mathrm{Wnt} / \beta$-catenin pathway, serum sclerostin levels, not DKK1, are positively correlated with cfPWV values in patients with hypertension. Meanwhile, serum sclerostin levels were found to be independent risk factors for the development of arterial stiffness in our patients.

\section{Abbreviations}

ACEi: Angiotensin-converting enzyme inhibitors; ARB: Angiotensin receptor blockers; BP: Blood pressures; BUN: Blood urea nitrogen; CCB: Calcium channel blockers; cfPW: Carotid-femoral pulse wave velocity; Cl: Confidence interval; CKD: Chronic kidney disease; CRIC: Chronic renal insufficiency cohort; DBP: Diastolic blood pressure; DKK1: Dickkopf-1; DM: Diabetes mellitus; eGFR: Estimated glomerular filtration rate; HDL-C: High-density lipoprotein 
cholesterol; iPTH: Intact parathyroid hormone; LDL-C: Low-density lipoprotein cholesterol; LRP5/6: Low-density lipoprotein receptor-related protein; PW: Pulse wave velocity; SBP: Systolic blood pressure; TCH: Total cholesterol; TG: Triglycerides

\section{Acknowledgements}

We thank Jen-Hung Wang (Department of Medical Research, Division of Epidemiology and Biostatistics Consulting Center, Buddhist Tzu Chi General Hospital, Hualien, Taiwan) for statistical consultation.

\section{Funding}

This work was supported by grants from Tzu Chi Hospital (TCRD100-02) in Taiwan.

\section{Availability of data and materials}

The datasets used and/or analysed during the current study are available from the corresponding author on reasonable request.

\section{Authors' contributions}

$\mathrm{BGH}$ and JHW conceived and designed the experiments. YCC, CJL, and JHW performed the experiments. CJL, BGH, and JHW contributed reagents and analyzed the data. YCC, HHL, and BGH wrote the manuscript. All of the authors reviewed and approved the final version of this paper.

\section{Ethics approval and consent to participate}

The study was approved by an independent ethics committee from the Protection of the Human Subjects Institutional Review Board of Tzu-Chi University and Hospital (IRB099-58), and all participants gave written informed consent according to the general recommendations of the Helsinki Declaration.

\section{Consent for publication}

All authors consent this manuscript for publication.

\section{Competing interests}

The authors declare that there is no conflict of interest regarding the publication of this paper.

\section{Publisher's Note}

Springer Nature remains neutral with regard to jurisdictional claims in published maps and institutional affiliations.

\section{Author details \\ ${ }^{1}$ School of Medicine, Tzu Chi University, Hualien, Taiwan. ${ }^{2}$ Division of Nephrology, Buddhist Tzu Chi General Hospital, Hualien, Taiwan. ${ }^{3}$ Division of Nephrology, Hsin-Jen Hospital, New Taipei City, Taiwan. ${ }^{4}$ Department of Nursing, Tzu Chi College of Technology, Hualien, Taiwan. ${ }^{5}$ Division of Cardiology, Buddhist Tzu Chi General Hospital, No. 707, Section 3, Chung-Yang Road, Hualien 97004, Taiwan.}

\section{Received: 18 June 2018 Accepted: 14 November 2018}

\section{Published online: 27 November 2018}

\section{References}

1. O'Shea PM, Griffin TP, Fitzgibbon M. Hypertension: the role of biochemistry in the diagnosis and management. Clin Chim Acta. 2017:465:131-43.

2. Vlachopoulos C, Aznaouridis K, Stefanadis C. Prediction of cardiovascular events and all-cause mortality with arterial stiffness: a systematic review and meta-analysis. J Am Coll Cardiol. 2010;55(13):1318-27.

3. Palatini P, Casiglia E, Gąsowski J, Głuszek J, Jankowski P, Narkiewicz K, Saladini F, Stolarz-Skrzypek K, Tikhonoff V, Van Bortel L, et al. Arterial stiffness, central hemodynamics, and cardiovascular risk in hypertension. Vasc Health Risk Manag. 2011;7:725-39.

4. Kubozono T, Ohishi M. Prognostic significance of regional arterial stiffness for stroke in hypertension. Pulse. 2015:3(2):98-105.

5. Humphrey JD, Harrison DG, Figueroa CA, Lacolley P, Laurent S. Central artery stiffness in hypertension and aging: a problem with cause and consequence. Circ Res. 2016;118(3):379-81.

6. Palombo C, Kozakova M. Arterial stiffness, atherosclerosis and cardiovascular risk: pathophysiologic mechanisms and emerging clinical indications. Vasc Pharmacol. 2016:77:1-7.

7. Pinto E. Blood pressure and ageing. Postgrad Med J. 2007;83(976):109-14.
8. Vlachopoulos C, Xaplanteris P, Aboyans V, Brodmann M, Cífková R, Cosentino F, De Carlo M, Gallino A, Landmesser U, Laurent S, et al. The role of vascular biomarkers for primary and secondary prevention. A position paper from the European Society of Cardiology Working Group on peripheral circulation: endorsed by the Association for Research into arterial structure and physiology (ARTERY) society. Atherosclerosis. 2015;241(2):507-32.

9. AlGhatrif M, Lakatta EG. The conundrum of arterial stiffness, elevated blood pressure. and aging Curr Hypertens Rep. 2015;17(2):12.

10. Mancia G, Fagard R, Narkiewicz K, Redón J, Zanchetti A, Böhm M, Christiaens T, Cifkova R, De Backer G, Dominiczak A, et al. ESH/ESC Guidelines for the management of arterial hypertension: the Task Force for the management of arterial hypertension of the European Society of Hypertension (ESH) and of the European Society of Cardiology (ESC). J Hypertens. 2013;31((10)):1925-38.

11. Lewiecki EM. Role of sclerostin in bone and cartilage and its potential as a therapeutic target in bone diseases. Ther Adv Musculoskelet Dis. 2014;6(2): $48-57$.

12. Cai $T$, Sun $D$, Duan $Y$, Wen $P$, Dai $C$, Yang J, He W. WNT/ $\beta$-catenin signalling promotes VSMCs to osteogenic transdifferentiation and calcification through directly modulating Runx2 gene expression. Exp Cell Res. 2016; 345(2):206-17.

13. Ma S, Yao S, Tian H, Jiao P, Yang N, Zhu P, Qin S. Pigment epitheliumderived factor alleviates endothelial injury by inhibiting Wnt/B-catenin pathway. Lipids Health Dis. 2017;16(1):31.

14. Zhou L, Liu Y. Wnt/B-catenin signalling and renin-angiotensin system in chronic kidney disease. Curr Opin Nephrol Hypertens. 2016;25(2):100-6.

15. Ke HZ, Richards WG, Li X, Ominsky MS. Sclerostin and Dickkopf-1 as therapeutic targets in bone diseases. Endocr Rev. 2012;33(5):747-83.

16. Evrard S, Delanaye P, Kamel S, Cristol JP, Cavalier E. SFBC/SN joined working group on vascular calcifications. Vascular calcification: from pathophysiology to biomarkers. Clin Chim Acta. 2015;438:401-14.

17. Claes KJ, Viaene L, Heye S, Meijers B, d'Haese P, Evenepoel P. Sclerostin: another vascular calcification inhibitor? J Clin Endocrinol Metab. 2013;98(8): 3221-8.

18. Hortells L, Sosa C, Guillén N, Lucea S, Millán Á, Sorribas V. Identifying early pathogenic events during vascular calcification in uremic rats. Kidney Int. 2017;92(6):1384-94.

19. Alberti KG, Zimmet PZ. Definition, diagnosis and classification of diabetes mellitus and its complications. I. Diagnosis and classification of diabetes mellitus: provisional report of a WHO consultation. Diabet Med. 1998;15(7): 539-53.

20. Hsu BG, Liou HH, Lee CJ, Chen YC, Ho GJ, Lee MC. Serum sclerostin as an independent marker of peripheral arterial stiffness in renal transplantation recipients-a cross-sectional study. Medicine. 2016;95(15):e3300.

21. Tsai JP, Wang JH, Chen ML, Yang CF, Chen YC, Hsu BG. Association of serum leptin levels with central arterial stiffness in coronary artery disease patients. BMC Cardiovasc Disord. 2016;16:80.

22. Wang JH, Lee $C J$, Yang CF, Chen YC, Hsu BG. Serum resistin as an independent marker of aortic stiffness in patients with coronary artery disease. PLoS One. 2017;12(8):e0183123.

23. Sun Z. Aging, arterial stiffness, and hypertension. Hypertension. 2015;65(2): 252-6.

24. Schram MT, Henry RM, van Dijk RA, Kostense PJ, Dekker JM, Nijpels G, Heine RJ, Bouter LM, Westerhof N, Stehouwer CD. Increased central artery stiffness in impaired glucose metabolism and type 2 diabetes: the Hoorn study. Hypertension. 2004;43(2):176-81.

25. Cecelja M, Chowienczyk P. Dissociation of aortic pulse wave velocity with risk factors for cardiovascular disease other than hypertension: a systematic review. Hypertension. 2009;54(6):1328-36.

26. Cheng YB, Li LH, Guo QH, Li FK, Huang QF, Sheng CS, Wang JG, Staessen JA, Li Y. Independent effects of blood pressure and parathyroid hormone on aortic pulse wave velocity in untreated Chinese patients. J Hypertens. 2017;35(9):1841-8

27. Woodard T, Sigurdsson S, Gotal JD, Torjesen AA, Inker LA, Aspelund T, Eiriksdottir G, Gudnason V, Harris TB, Launer $\sqcup$, et al. Mediation analysis of aortic stiffness and renal microvascular function. J Am Soc Nephrol. 2015;26(5):1181-7.

28. Townsend RR, Wimmer NJ, Chirinos JA, Parsa A, Weir M, Perumal K, Lash JP, Chen J, Steigerwalt SP, Flack J, et al. Aortic PWV in chronic kidney disease: a CRIC ancillary study. Am J Hypertens. 2010;23(3):282-9.

29. Garnier AS, Briet M. Arterial stiffness and chronic kidney disease. Pulse. 2016; 3(3-4):229-41. 
30. Besler C, Lüscher TF, Landmesser U. Molecular mechanisms of vascular effects of high-density lipoprotein: alterations in cardiovascular disease. EMBO Mol Med. 2012;4(4):251-68.

31. Riwanto M, Landmesser U. High density lipoproteins and endothelial functions: mechanistic insights and alterations in cardiovascular disease. J Lipid Res. 2013;54(12):3227-43.

32. Wang F, Ye P, Luo L, Xiao W, Qi L, Bian S, Wu H, Sheng L, Xiao T, Xu R. Association of serum lipids with arterial stiffness in a population-based study in Beijing. Eur J Clin Investig. 2011;41(9):929-36.

33. Wang X, Du Y, Fan L, Ye P, Yuan Y, Lu X, Wang F, Zeng Q. Relationships between HDL-C, hs-CRP, with central arterial stiffness in apparently healthy people undergoing a general health examination. PLoS One. 2013;8(12): e81778.

34. Peiris AN, Youssef D, Grant WB. Secondary hyperparathyroidism: benign bystander or culpable contributor to adverse health outcomes? South Med J. 2012;105(1):36-42.

35. Hagström $E$, Michaëlsson $K$, Melhus $H$, Hansen $T$, Ahlström $H$, Johansson L, Ingelsson E, Sundström J, Lind L, Arnlöv J. Plasma-parathyroid hormone is associated with subclinical and clinical atherosclerotic disease in 2 communitybased cohorts. Arterioscler Thromb Vasc Biol. 2014;34(7):1567-73.

36. Tölle M, Reshetnik A, Schuchardt M, Höhne M, van der Giet M. Arteriosclerosis and vascular calcification: causes, clinical assessment and therapy. Eur J Clin Invest. 2015;45(9):976-85.

37. Pikilidou M, Yavropoulou M, Antoniou M, Yovos J. The contribution of osteoprogenitor cells to arterial stiffness and hypertension. J Vasc Res. 2015; 52(1):32-40.

38. Zhu D, Mackenzie NC, Millán JL, Farquharson C, MacRae VE. The appearance and modulation of osteocyte marker expression during calcification of vascular smooth muscle cells. PLoS One. 2011;6(5):e19595.

39. Sehgel NL, Vatner SF, Meininger GA. "Smooth muscle cell stiffness syndrome"-revisiting the structural basis of arterial stiffness. Front Physiol. 2015;6:335.

40. Kirkpantur A, Balci M, Turkvatan A, Afsar B. Independent association between serum sclerostin levels and carotid artery atherosclerosis in prevalent haemodialysis patients. Clin Kidney J. 2015;8(6):737-43.

41. Morena M, Jaussent I, Dupuy AM, Bargnoux AS, Kuster N, Chenine L, LerayMoragues H, Klouche K, Vernhet H, Canaud B, et al. Osteoprotegerin and sclerostin in chronic kidney disease prior to dialysis: potential partners in vascular calcifications. Nephrol Dial Transplant. 2015;30(8):1345-56.

42. Wang XR, Yuan L, Zhang JJ, Hao L, Wang DG. Serum sclerostin values are associated with abdominal aortic calcification and predict cardiovascular events in patients with chronic kidney disease stages 3-5D. Nephrology. 2017;22(4):286-92.

43. Desjardins L, Liabeuf S, Oliveira RB, Louvet L, Kamel S, Lemke HD, Vanholder R, Choukroun G, Massy ZA. European Uremic Toxin (EUTox) Work Group. Uremic toxicity and sclerostin in chronic kidney disease patients. Nephrol Ther. 2014;10(6):463-70.

44. Jin S, Zhu M, Yan J, Fang Y, Lu R, Zhang W, Zhang Q, Lu J, Qi C, Shao X, et al. Serum sclerostin level might be a potential biomarker for arterial stiffness in prevalent hemodialysis patients. Biomark Med. 2016;10(7):689-99.

45. Hampson G, Edwards S, Conroy S, Blake GM, Fogelman I, Frost ML. The relationship between inhibitors of the Wnt signaling pathway (Dickkopf1(DKK1) and sclerostin), bone mineral density, vascular calcification and arterial stiffness in post-menopausal women. Bone. 2013;56(1):42-7.

46. Morales-Santana S, García-Fontana B, García-Martín A, Rozas-Moreno P, García-Salcedo JA, Reyes-García R, Muñoz-Torres M. Atherosclerotic disease in type 2 diabetes is associated with an increase in sclerostin levels. Diabetes Care. 2013;36(6):1667-74.

47. Hruska KA, Sugatani T, Agapova O, Fang Y. The chronic kidney disease mineral bone disorder (CKD-MBD): advances in pathophysiology. Bone. 2017:100:80-6.

48. Gaudio A, Fiore V, Rapisarda R, Sidoti MH, Xourafa A, Catalano A, Tringali G, Zanoli L, Signorelli SS, Fiore CE. Sclerostin is a possible candidate marker of arterial stiffness: results from a cohort study in Catania. Mol Med Rep. 2017; 15(5):3420-4.

\section{Ready to submit your research? Choose BMC and benefit from:}

- fast, convenient online submission

- thorough peer review by experienced researchers in your field

- rapid publication on acceptance

- support for research data, including large and complex data types

- gold Open Access which fosters wider collaboration and increased citations

- maximum visibility for your research: over $100 \mathrm{M}$ website views per year

At BMC, research is always in progress.

Learn more biomedcentral.com/submissions 